\title{
Molecular Targeted Drug Therapy of Ovarian Cancer
}

\author{
Lili Fan, Yang Shen*, Chen Xi, Mulan Ren \\ Department of Obstetrics and Gynaecology, Zhongda Hospital, School of Medicine \\ Southeast University, Nanjing 210009, China \\ "Department of Obstetrics and Gynecology, Zhongda Hospital, Southeast University, Nanjing 210009 \\ shenyang0924@sina.cn
}

\begin{abstract}
Molecular targeted therapy as a new means of cancer therapy targeting molecular pathological abnormalities of cancer, the use of target binding ligand and receptor can effectively improve drug lethal to tumor cells while reducing adverse effects on normal tissues and organs, it has been gradually used in clinical treatment. In the treatment of ovarian cancer in comparison with conventional treatment modalities molecular targeted therapy showed significant advantages. In this paper from monoclonal antibodies, signal pathway inhibitors for use both terms of targeted therapy in ovarian cancer treatment and prospects to Summary and Prospects.
\end{abstract}

Keywords: ovarian cancer, molecular targeted therapy, monoclonal antibodies, signal pathway inhibitors.

In the female genital malignancies, ovarian cancer incidence rate in the first three, mortality ranking the first bit $^{[1]}$.Ovaries deep in the pelvis, due to the lack of effective and accurate method for early diagnosis, about $70 \%$ to $80 \%$ of patients once diagnosed advanced stage. Although cytoreductive surgery plus platinum or paclitaxel technology has improved, the recurrence rate is still as high as $85 \%$ in patients with advanced ovarian cancer 5-year survival rate is still hovering around $20 \%$ to $30 \%{ }^{[2]}$. In recent years, cancer targeted therapy is booming. The so-called targeted therapy refers to the use of tumor tissue is different from normal tissue characteristics, given targeted treatment, so as to achieve a better therapeutic effect and minimize adverse reactions ${ }^{[3,4]}$. With the development of medical theory and techniques of molecular biology, targeted therapy has become a new trend of treatment of ovarian cancer.

Currently molecules for the treatment of ovarian cancer targeted drugs based on targets and different mechanism of action, divided into: (1) anti-angiogenesis inhibitor ${ }^{[5]}$, a recombinant humanized anti-vascular endothelial growth factor (VEGF) monoclonal antibodies such as Avastin mAb (BEV); against VEGF receptor tyrosine kinase inhibitors such as sorafenib, pazopanib; angiogenesis inhibitors such as Trebananib. (2) epidermal growth factor receptor (EGFR) inhibitors ${ }^{[6]}$, monoclonal antibodies such as anti-EGFRs trastuzumab, cetuximab, etc; for the EGFR tyrosine kinase inhibitors such as gefitinib, Heureaux for Nigeria and so on. (3) adenosine diphosphate ribose polymerase (PARP) inhibitors ${ }^{[7]}$, such as Ola Trapani, Veliparib like. (4) platelet-derived derived factor receptor (PDGFR) inhibitors ${ }^{[8]}$, such as imatinib and so on. (5) Other drugs, such as small-molecule inhibitor of metalloproteinase Shali Du amine, PI3k / Akt / mTOR pathway inhibitors such as according to Wei $\operatorname{Mosi}^{[9]}$, for sirolimus, folate receptor inhibitors, such as pemetrexed, Vintafolide cyclooxygenase -1 (COX-1) inhibitors and so on.

\subsection{Application of Bevacizumab in Ovarian Cancer}

VEGF expression level in ovarian cancer tissues was significantly higher than in normal ovarian tissue, VEGF titer is closely related to prognosis and staging of tumors, VEGF is an independent prognostic factor of ovarian cancer ${ }^{[10]}$. Anti-angiogenic drugs $\mathrm{BEV}$ is the most widely used in gynecologic cancer research and clinical applications. BEV is the first to be generated by the US Food and Drug Administration (FDA) approved anti-angiogenic antibodies. 
Application BEV in line chemotherapy as well as maintenance therapy after first-line chemotherapy can achieve significant survival benefit. Twenty-five patients with high-grade serous AOC treated with bevacizumab-based NACT (cases) were matched according to initial disease extension assessed by laparoscopy, and age, in a 1:2 ratio, with 50 high-grade serous AOC patients treated with standard NACT without bevacizumab (controls). Both groups received a median of four NACT cycles before IDS $(\mathrm{p}=0.867)$, and the median time interval between NACT and IDS was 27 days in both groups ( $\mathrm{p}$ $=0.547)$. Twenty-two cases $(88.0 \%)$ showed complete/partial radiologic response compared with 36 controls $(72.3 \% ; \mathrm{p}=0.054)$. A higher percentage of cases showed complete serological response (48 vs. $35.1 \% ; \mathrm{p}=0.041)$. At IDS, complete cytoreduction was achieved in 20 cases $(80.0 \%)$ and 36 controls $(72.3 \%)$ [ $\mathrm{p}=0.260]$. Cases showed a longer median PFS compared with controls (18 months vs. 10 months; $p=0.001$ ), and the administration of bevacizumab (hazard ratio $3.786 ; p=0.001$ ) retained a prognostic role for longer PFS at multivariate analysis ${ }^{[11]}$.

\subsection{Application of Pazopanib in Ovarian Cancer}

Pazopanib is a second-generation multitargeted tyrosine kinase inhibitor against vascular endothelial growth factor receptor-1,-2, and-3, platelet-derived growth factor receptor- $\alpha$, platelet-derived growth factor receptor- $\beta$, and c-kit. Preclinical evaluation has revealed excellent antiangiogenic and antitumor activity, and synergism was observed in combination with chemotherapeutic drugs ${ }^{[2,13]}$.

A study is designed as a multicenter phase I/II trial evaluating the optimal dose for pazopanib (phase I) as well as activity and tolerability of a combination regimen consisting of pazopanib and metronomic cyclophosphamide in the palliative treatment of patients with recurrent, platinum-resistant, pre-treated ovarian cancer (phase II). The current phase I/II trial shall clarify the potential of the multitargeting antiangiogenic tyrosinkinase inhibitor GW 786034 (pazopanib) in combination with oral cyclophosphamide as salvage treatment in patients with recurrent, pretreated ovarian cancer ${ }^{[14]}$.

\subsection{Application of Trastuzumab in Ovarian Cancer}

HER-2 is a transmembrane tyrosine kinase receptor similar to epidermal growth factor receptor (EGFR). At present, most studies have found high expression of HER-2 with poor prognosis in ovarian cancer ${ }^{[15]}$. Trastuzumab (Herceptin) is a humanized monoclonal HER-2 antibody, which can be combined with the extracellular domain of the HER-2 and inhibit growth of tumor which have high expression of HER-2 and enhance anti-tumor activity effect of cell poison drugs. Given the recent success of EGFR-targeted therapeutics for the treatment of other solid tumors, and the well-established safety profile of trastuzumab, results presented here provide a rationale for re-evaluation of trastuzumab as an experimental ovarian cancer therapeutic, either in concert with, or perhaps as a "primer" for EGFR targeted therapeutics ${ }^{[16]}$.

\subsection{Application of Gefitinib in Ovarian Cancer}

Gefitinib (Iressa) is an oral aniline quinoline compounds that can inhibit EGFR tyrosine kinase blocking signal transduction, blocking cell proliferation signal transduction pathways, reducing angiogenesis, which currently treatment has been approved by the FDA for non-small cell lung cancer ${ }^{[17]}$. In vitro, gefitinib can inhibit the growth of human ovarian cancer cell lines.

Pautier $\mathrm{P}^{\prime} \mathrm{s}^{[18]}$ team reported Phase II clinical trial results of gefitinib combined with paclitaxel, platinum as second-line treatment of ovarian cancer, fallopian tube cancer and peritoneal cancer. 69 cases of patients ( 26 cases of platinum-resistant and platinum-sensitive 42 cases), platinum-resistant overall efficiency and disease control rate was $19.2 \%$ and $69.2 \%$ respectively, platinum-sensitive persons were $61.9 \%$ and $81.0 \%$.

\section{APPLICATION OF PARP INHIBITORS IN OVARIAN CANCER}

Poly(ADP-ribose) polymerase (PARP) is an abundant nuclear enzyme, which is recruited to repair DNA damage in cells, and also plays a role in cell proliferation, differentiation and transformation ${ }^{[19]}$. Ola Jesper (Olaparib) is an oral PARP inhibitor used in the clinical treatment of ovarian cancer. A phase II clinical application Ola Trapani treatment exists BRCA mutations platinum-sensitive or 
platinum-resistant ovarian cancer patients showed that the overall objective response rate was $40 \%$, platinum sensitive and platinum-resistant, clinical platinum refractory group has efficiency of $69 \%$, $45 \%$ and $23 \%$ respectively ${ }^{[20]}$. It concluded that for BRCA1 / 2 mutation ovarian cancer Ola Trapani has anti-tumor effects, clinical benefit associated with platinum sensitivity. Another clinical trial phase II selected cancer patients with recurrent ovarian BRCA mutation, were given $400 \mathrm{mg}$ Ola Trapani and $100 \mathrm{mg}, 2$ times / d, showed objective response rate was 33\% and 13\%, respectively, higher doses group better efficacy ${ }^{[21]}$.

\section{APPLICATION OF PDGFR INHIBITORS IN OVARIAN CANCER}

PDGF can induce cell growth, transformation, migration, vascular permeability, and wound healing. PDGFR is a connective tissue between the media, it is also a key factor in tumorigenesis ${ }^{[22]}$. PDGFR targeted drugs, including imatinib ${ }^{[23]}$ as well as other multi-target TKIs, such as: gefitinib, sorafenib, etc. (mentioned before). In addition, PDGFR-specific monoclonal antibody is also reported, IMC-3G3 and MEDI-575 has separately entered II and I clinical trials, their efficacy is worth looking forward to ${ }^{[24]}$. Imatinib is a selective inhibitor of Abl, c-Kit and PDGFR, since the protein structure and gene mapping of PDGFR are similar to c-Kit, the two often closely related. Imatinib has been widely used to treat leukemia and gastrointestinal stromal tumors, and achieved very good clinical results. Ovarian cancer is highly expressed c-Kit and (or) PDGFR, they play an important role in the development in ovarian cancer. Vitro experiments confirmed that imatinib can prevent the growth of ovarian cancer cell lines, and change the apoptosis, signal transduction. However, II clinical trials ${ }^{[23]}$ have shown that, although patients can better tolerate imatinib, but no significant effect, and thus limited the application of ovarian cancer. Nevertheless, there is still a need for further study whether imatinib as chemotherapy or radiation sensitizer to reduce the dose of chemotherapy to reduce adverse reactions, delayed resistance, thereby improving survival and quality of life of patients.

\subsection{Application of Thalidomide in Ovarian Cancer}

Thalidomide is an oral immunomodulatory agent with antiangiogenic properties and activity in ovarian cancer $^{[25]}$. It inhibits TNF-beta production in lipopolysaccharide-stimulated monocytes. Thalidomide decreased significantly the capacity of SKOV-3 cells and primary epithelial ovarian carcinoma cells to secrete TNF-alpha, it also significantly decreased the capacity of SKOV-3 cells, but not primary epithelial ovarian carcinoma cells, to secrete MMP-9 and MMP-2. The results have important implications in future therapeutic strategies that will incorporate thalidomide and other cytokine inhibitors in the treatment of epithelial ovarian carcinoma ${ }^{[26]}$.

\subsection{Application of Signal Pathway Inhibitors in Ovarian Cancer}

There are several signal transduction pathways in tumor cells control system, abnormal expression of signaling pathways closely associated with tumor growth, invasion, metastasis, angiogenesis, radiotherapy, chemotherapy resistance and other characteristics are also related ${ }^{[27]}$. There are three main ways: Ras / Raf / mitogen-activated protein kinase kinase / wire mitogen-activated protein kinase (Ras / Raf / MEK / MAPK) pathway, phosphatidylinositol 3-kinase / protein kinase C / IkB kinase (PI3K / PKC / IKK) pathway, JAK kinase / signal transducer activators of transcription (JAK / STAT) pathway ${ }^{[28]}$. At present, for targeting these pathways drugs which are constantly entering clinical trials.

\subsubsection{Application of RAs/RAF/MAP Pathway Inhibitors in Ovarian Cancer}

The RAS/RAF/MAP kinase--ERK kinase (MEK)/extracellular-signal-regulated kinase (ERK) (MAPK) pathways are frequently deregulated in human cancer as a result of genetic alterations in their components or upstream activation of cell-surface receptors. These signalling cascades are regulated by complex feedback and cross-talk mechanisms ${ }^{[29]}$. R115777 is a small molecule farnesyl transferase inhibitors, can also inhibit the RAS signaling pathway and P13K-AKT signaling pathway. Stacey A. Taylor $^{[30]}$ found that the combination of lonafarnib plus paclitaxel resulted in marked tumor regressions in A2780, TOV-112D, PA-1, and IGROV-1 tumor xenografts. Lonafarnib enhances the antiproliferative effects of paclitaxel on ovarian cancer cells in vitro and ovarian tumor xenografts in vivo. 
Lapatinib is an oral small molecule tyrosine kinase inhibitor, it can inhibit the MAPK activation, thereby inhibiting cell proliferation. Lapatinib can also inhibit the activation of AKT and leads to increased tumor cell apoptosis. Phase I clinical studies of lapatinib plus carboplatin for platinum-sensitive recurrent ovarian cancer showed the 11 patients had a partial response in three, three stable disease, major toxicities were diarrhea, rash, nausea, fatigue ${ }^{[31]}$. Weroha $\mathrm{SJ}^{[32]}$ studied phase II clinical trials of lapatinib Nigeria and topotecan treatment of platinum-resistant ovarian cancer and primary peritoneal cancer in 18 patients, only one case partially valid, three cases in stable condition. Although in this study the effect is not significant, but the GOG continues to study Phase II clinical for the treatment of advanced ovarian cancer.

\subsubsection{Application of PI3K / PKC / IKK pathway Inhibitors in Ovarian Cancer}

PI3K / AKT signaling pathways have a great relationship with cell survival, growth, transformation and tumor formation. In recent years, signaling pathways and tumor molecular targeted therapy has become a hot research.

Weberpals ${ }^{[33]}$ found that inhibition of phosphorylation of Akt in human ovarian cancer cell lines SKOV3 and PA-1, the taxol-induced apoptosis increased. Application of specific PI3K-Akt inhibitors (LY294002) synergistically increase the effectiveness of paclitaxel-induced apoptosis. mTOR inhibitor temsirolimus and everolimus can inhibit tumor growth, angiogenesis, and tumor invasion and can enhance the expression of paclitaxel-induced Akt / mTOR apoptosis in ovarian cancer cells ${ }^{[34]}$. It has been confirmed in planting model of ovarian cancer. Some clinical trials found that while targeting VEGF and mTOR therapy can improve the anti-cancer effect.

Aprinocarsen PKC is an antisense nucleic acid, which can specifically bind and degrade mRNA of PKC, thereby inhibiting the expression and function of PKC. A II clinical study from Advani reported that 36 patients with advanced ovarian cancer patients were divided into platinum-sensitive and platinum-resistant group, group were given single-drug Aprinoearsen treatment, only one case of drug-resistant patients with decreased levels of serum CAl25 and in a stable condition up to eight months, overall no significant relief ${ }^{[35]}$.

\subsubsection{Application of JAK / STAT Pathway Inhibitors in Ovarian Cancer}

Interaction between tumor cells and their microenvironment has a crucial role in the development, progression and drug resistance of cancer. Soluble factors secreted by Hospicells activate several genes and upregulate the JAK/STAT signaling pathway in ovarian cancer cell lines. IGF-I is a major regulator of different stages of cancer development. It regulates the expression of $\mathrm{ABC}$ genes in OVCAR3 cells via the PI3-kinase, MEK and JAK2/STAT3 signaling pathways. The OVCAR3 cell line when co-cultured with Hospicells showed a marked degree of drug resistance. The drug resistance observed could be amplified with exogenous IGF-I. Addition of IGF-IR inhibitor, however, reduced the degree of resistance in these exposed cells. So it concluded that the inhibition of IGF-IR and targeting of the JAK2/STAT3 signaling pathway can be a target for ovarian cancer therapy ${ }^{[36]}$.

\section{APPLICATION OF FOLATE RECEPTOR INHIBITORS IN OVARIAN CANCER}

In recent years, we found that in 90 percent of ovarian cancer cells over expressed folate receptors, the body's immune response to folic acid is relatively small, therefore the study of folic acid as a carrier targeted delivery of drugs to treat ovarian cancer have great significance ${ }^{[37]}$. Nukolova ${ }^{[38]}$ studied the use of nano-gel wrapped drugs with folic acid as a carrier to treat ovarian cancer, folic acid nanogels drugs inhibit peritoneal ovarian xenograft tumor growth apparently, the treatment effect of using folate carrier is better than no use, and also there is no toxicity to normal tissue cells. So folic acid nanogels drugs targeted delivery of anti-cancer drugs, not only enhances the therapeutic relevance, but also reduce the systemic toxicity of conventional therapy.

\section{APplication OF COX INHIBITORS IN OVARIAN CANCER}

Cyclooxygenase-1 (COX-1) is expressed at high levels in the early stages of human epithelial ovarian cancer where it seems to play a key role in cancer onset and progression. As a consequence, COX-1 is 
an ideal biomarker for early ovarian cancer detection ${ }^{[39]}$. Paola Vitale ${ }^{[40]}$ reported suggest some molecular determinants necessary to design new diarylisoxazoles as inhibitors of cyclooxygenases, mainly COX-1.COX-1 inhibitor can be used as anti-inflammatories, anti proliferative and chemopreventive drugs.

Celecoxib, a highly selective cyclooxygenase-2 inhibitor, regulates apoptosis of several types of human cancer cells. $\mathrm{Kim}^{[41]}$ reported that OVCAR-3 cells were sensitized to paclitaxel-induced apoptosis by celecoxib through downregulation of NF-kappa B and Akt activation, suggesting that celecoxib may work synergistically with paclitaxel to inhibit different targets and ultimately produce anticancer effects. Combining celecoxib with paclitaxel may prove beneficial in the clinical treatment of ovarian cancer.

Targeted treatment of ovarian cancer with high relevance and prospects are very bright, but it is still in the preliminary study stage, there are many issues to be resolved, such as: molecular targeted therapy targeting the poor, more murine-derived antibody heterogeneity, mutation and tumor signal transduction compensatory may cause resistance, time and order of administration and the like. In addition to molecular targeted therapy, targeted gene therapy topic worth further exploring. Ovarian cancer occurrence and development is the result of a multi-target multi-link control and different histopathological types of ovarian cancer have different molecular abnormalities mechanism. Therefore, we need a better understanding of the key mechanisms of ovarian cancer cancerous abnormal molecules, discovered more critical targets in a complex signal path, and depending on the subtype of tumor molecular pathology, choose valuable targeted therapy.

\section{REFERENCES}

[1] Kigawa J. Molecular-targeted therapies for ovarian cancer[J]. Int J Clin Oncol. 2012,17(5):423-.

[2] Gabra H. Introduction to managing patients with recurrent ovarian cancer[J]. European Journal of Cancer Supplements. 2014,12(2):2-6.

[3] Tang MK, Wong AS. Exosomes: Emerging biomarkers and targets for ovarian cancer[J]. Cancer letters. 2015,367(1):26-33.

[4] Zhang X, Yuan X, Shi H, Wu L, Qian H, Xu W. Exosomes in cancer: small particle, big player[J]. Journal of hematology \& oncology. 2015,8:83.

[5] Li J, Li S, Chen R, Yu H, Lu X. The prognostic significance of anti-angiogenesis therapy in ovarian cancer: a meta-analysis[J]. Journal of ovarian research. 2015,8:54.

[6] AWilken1 J, TWebster K, JMaihle N. Research Trastuzumab Sensitizes Ovarian Cancer Cells to EGFR-targeted Therapeutics[J]. Journal of ovarian research. 2010,3(7):1-9.

[7] Chen Y, Zhang L, Hao Q. Olaparib: a promising PARP inhibitor in ovarian cancer therapy[J]. Archives of gynecology and obstetrics. 2013,288(2):367-74.

[8] Werner TL, Wade ML, Agarwal N, Boucher K, Patel J, Luebke A, et al. A pilot study of JI-101, an inhibitor of VEGFR-2, PDGFR-beta, and EphB4 receptors, in combination with everolimus and as a single agent in an ovarian cancer expansion cohort[J]. Investigational new drugs. 2015.

[9] Li H, Zeng J, Shen K. PI3K/AKT/mTOR signaling pathway as a therapeutic target for ovarian cancer[J]. Archives of gynecology and obstetrics. 2014,290(6):1067-78.

[10] Smerdel MP, Steffensen KD, Waldstrøm M, Andersen RF, Olsen DA, Brandslund I, et al. VEGF in the Development of Ovarian Malignancy[J]. Clinical Ovarian Cancer. 2011,4(1):19-25.

[11] Petrillo M, Paris I, Vizzielli G, Amadio G, Cosentino F, Salutari V, et al. Neoadjuvant Chemotherapy Followed by Maintenance Therapy With or Without Bevacizumab in Unresectable High-Grade Serous Ovarian Cancer: A Case-Control Study[J]. Annals of surgical oncology. 2015. 
[12] Sonpavde G, Hutson TE, PharmD. Pazopanib: A Novel Multitargeted Tyrosine Kinase Inhibitor[J]. Current Oncology Reports. 2007,9(2):115-9.

[13] Jackson AL, Eisenhauer EL, Herzog TJ. Emerging therapies: angiogenesis inhibitors for ovarian cancer[J]. Expert opinion on emerging drugs. 2015,20(2):331-46.

[14] Eichbaum M, Mayer C, Eickhoff R, Bischofs E, Gebauer G, Fehm T. The PACOVAR-trial: A phase I/II study of pazopanib (GW786034) and cyclophosphamide in patients with platinum-resistant recurrent,pre-treated ovarian cancer[J]. BMC Cancer. 2011,11:453-62.

[15] McAlpine J, Wiegand K, Miller M, Adamiak A, Koebel M, Vang R, et al. HER2 Overexpression and amplification is present in a subset of ovarian mucinous carcinomas and can be targeted with trastuzumab therapy[J]. Gynecologic Oncology. 2010,116(3):593-4.

[16] AWilken J, TWebster K, JMaihle N. Research Trastuzumab Sensitizes Ovarian Cancer Cells to EGFR-targeted Therapeutics[J]. Journal of ovarian research. 2010,3(7):1-9.

[17] Sugita S, Ito K, Yamashiro Y, Moriya S, Che X-F, Yokoyama T, et al. EGFR-independent autophagy induction with gefitinib and enhancement of its cytotoxic effect by targeting autophagy with clarithromycin in non-small cell lung cancer cells[J]. Biochemical and Biophysical Research Communications. 2015,461(1):28-34.

[18] Yoshimura N, Kudoh S, Mitsuoka S, Yoshimoto N, Oka T, Nakai T, et al. Phase II study of a combination regimen of gefitinib and pemetrexed as first-line treatment in patients with advanced non-small cell lung cancer harboring a sensitive EGFR mutation[J]. Lung Cancer. 2015,90(1):65-70.

[19] Tangutoori S, Baldwin P, Sridhar S. PARP inhibitors: A new era of targeted therapy[J]. Maturitas. 2015,81(1):5-9.

[20] Fong PC, Yap TA, Boss DS, Carden CP, Mergui-Roelvink M. Poly(ADP)-ribose polymerase inhibition: frequent durable responses in BRCA carrier ovarian cancer correlating with platinum-free interval[J]. Journal of clinical oncology. 2010,28(15):2512-9.

[21] Audeh MW, Carmichael J, Penson RT, Friedlander M, Powell B. Oral poly(ADP-ribose) polymerase inhibitor olaparib in patients with BRCA1 or BRCA2 mutations and recurrent ovarian cancer: a proof-of-concept trial[J]. The Lancet. 2010,376(9737):245-51.

[22] Apte SM, Bucana CD, Killion JJ, Gershenson DM, Fidler IJ. Expression of platelet-derived growth factor and activated receptor in clinical specimens of epithelial ovarian cancer and ovarian carcinoma cell lines[J]. Gynecologic Oncology. 2004,93(1):78-86.

[23] Coleman RL, Broaddus RR, Bodurka DC, Wolf JK, Burke TW, Kavanagh JJ, et al. Phase II trial of imatinib mesylate in patients with recurrent platinum- and taxane-resistant epithelial ovarian and primary peritoneal cancers[J]. Gynecologic Oncology. 2006,101(1):126-31.

[24] Shah GD, Loizos N, Youssoufian H, Schwartz JD, Rowinsky EK. Rationale for the development of IMC-3G3, a fully human immunoglobulin G subclass 1 monoclonal antibody targeting the platelet-derived growth factor receptor alpha[J]. Cancer. 2010,116(4 Suppl):1018-26.

[25] Buttin BM, Moore MJ. Thalidomide-induced reversible interstitial pneumonitis in a patient with recurrent ovarian cancer[J]. Gynecologic Oncology. 2008,111(3):546-8.

[26] Piura B, Medina L, Rabinovich A, Dyomin V, Huleihel M. Thalidomide distinctly affected TNF- $\alpha$, IL-6 and MMP secretion by an ovarian cancer cell line (SKOV-3) and primary ovarian cancer cells[J]. European cytokine network. 2013,24(3):122-9.

[27] Curry E, Cheraghchi-Bashi-Astaneh A, Chen M, Cunnea P, Camila DS, editors. DNA-PKcs is amplified in high-grade serous ovarian cancer (HGSC), correlates with poor outcome and drives resistance to platinum therapy via the AKT signaling pathway2015: American Association for Cancer Research. 
[28] Ptak A, Gregoraszczuk EL. Bisphenol A induces leptin receptor expression, creating more binding sites for leptin, and activates the JAK/Stat, MAPK/ERK and PI3K/Akt signalling pathways in human ovarian cancer cell[J]. Toxicology Letters. 2012,210(3):332-7.

[29] De Luca A, Maiello MR, D'Alessio A, Pergameno M, Normanno N. The RAS/RAF/MEK/ERK and the PI3K/AKT signalling pathways: role in cancer pathogenesis and implications for therapeutic approaches[J]. Expert opinion on therapeutic targets. 2012,16 Suppl 2(s2):S17-S27.

[30] Taylor SA, Marrinan CH, Liu G, Nale L, Bishop WR, Kirschmeier P, et al. Combining the farnesyltransferase inhibitor lonafarnib with paclitaxel results in enhanced growth inhibitory effects on human ovarian cancer models in vitro and in vivo[J]. Gynecologic Oncology. 2008,109(1):97-106.

[31] Kimball KJ, Numnum TM, Kirby TO, Zamboni WC, Estes JM, Barnes MN, et al. A phase I study of lapatinib in combination with carboplatin in women with platinum sensitive recurrent ovarian carcinoma[J]. Gynecologic Oncology. 2008,111(1):95-101.

[32] Weroha SJ, Oberg AL, Ziegler KLA, Dakhilm SR, Rowland KM, Hartmann LC, et al. Phase II trial of lapatinib and topotecan (LapTop) in patients with platinum-refractory/resistant ovarian and primary peritoneal carcinoma[J]. Gynecologic Oncology. 2011,122(1):116-20.

[33] Weberpals JI, Koti M, Squire JA. Targeting genetic and epigenetic alterations in the treatment of serous ovarian cancer[J]. Cancer genetics. 2011,204(10):525-35.

[34] Dinh P, Harnett P, Piccart-Gebhart MJ, Awada A. New therapies for ovarian cancer: Cytotoxics and molecularly targeted agents[J]. Critical Reviews in Oncology/Hematology. 2008,67(2):103-12.

[35] Advani R, Peethambaram P, Lum BL, Fisher GA, Hartmann L, Long HJ, et al. A Phase II trial of aprinocarsen, an antisense oligonucleotide inhibitor of protein kinase $\mathrm{C}$ alpha, administered as a 21-day infusion to patients with advanced ovarian carcinoma[J]. Cancer. 2004,100(2):321-6.

[36] Benabbou N, Mirshahi P, Cadillon M, Soria J, Therwath A. Hospicells promote upregulation of the ATP-binding cassette genes by insulin-like growth factor-I via the JAK2/STAT3 signaling pathway in an ovarian cancer cell line[J]. International journal of oncology. 2013,43(3):685-94.

[37] Bauerschlag DO, Maass N, Leonhardt P, Verburg FA, Pecks U, Zeppernick F, et al. Fatty acid synthase overexpression: target for therapy and reversal of chemoresistance in ovarian cancer[J]. Journal of translational medicine. 2015,13:146.

[38] Nukolova NV, Oberoi HS, Cohen SM, Kabanov AV, Bronich TK. Folate-decorated nanogels for targeted therapy of ovarian cancer[J]. Biomaterials. 2011,32(23):5417-26.

[39] Perrone MG, Malerba P, Uddin MJ, Vitale P, Panella A, Crews BC, et al. PET radiotracer [18F]-P6 selectively targeting COX-1 as a novel biomarker in ovarian cancer: Preliminary investigation[J]. European Journal of Medicinal Chemistry. 2014,80:562-8.

[40] Vitale P, Perrone MG, Malerba P, Lavecchia A, Scilimati A. Selective COX-1 inhibition as a target of theranostic novel diarylisoxazoles[J]. European Journal of Medicinal Chemistry. 2014,74:606-18.

[41] Kim HJ, Yim GW, Nam EJ, Kim YT. Synergistic Effect of COX-2 Inhibitor on Paclitaxel-Induced Apoptosis in the Human Ovarian Cancer Cell Line OVCAR-3[J]. Cancer research and treatment. 2014,46(1):81-92. 